\title{
Review of: "Functional and molecular characterization of a non-human primate model of autism spectrum disorder shows similarity with the human disease"
}

\author{
Minoo Rassoulzadegan ${ }^{1}$ \\ 1 Université de Nice-Sophia Antipolis
}

Potential competing interests: The author(s) declared that no potential competing interests exist.

Review of: Functional and molecular characterization of a non-human primate model of autism spectrum disorder shows similarity with the human disease

Autism is a very complex brain disorder with widely varying expression among patients presumably associated with multiple etiologic factors. The authors report a non-human primate model of ASD, attempting to relate similarities to the characteristics observed in human ASD. They analyze in three age groups the functional and molecular characterization of autism induced by valproic acid (VPA) in marmosets.

Pregnant women exposure to antiepileptic effect of VPA increases risk to give birth to children with autism by enhancing $Y$-aminobutyric acid (GABA) functions (Gould et al.2004; Kwan et al.2001). Dose dependant studies with VPA on rodent models confirmed its general effect on brain development. VPA is also known as teratogen (Eadie, 2008). VPA indirectly inhibits histone deacetylase (HDAC) (Phiel et al.2001), and is classified as an inhibitor against class I HDAC families (Kazantsev \& Thompson, 2008).

First, the rationale for proposing a new animal model of autism is very valuable. Finding conditions in the marmoset that coincide with selected human ASDs samples is remarkable work. This is true for functional and histological analysis. The results presented in 'Fig. 3 Altered isolation calls in VPA-exposed marmosets` are very important and convincing. Second, this study is important to highlight and especially confirm other studies (some recent references on mice are missing) on the critical importance of early markers for detection of ASD and the development of future therapies.

However, it is strange that they are arguing for a better model for human ASDs when all animal models are known to be complementary. Their working hypothesis is still based on a strong presupposed assertion that rodents are not good models for autism! Because, all autism occurrences in human are not induced by VPA and also in models (rat / or mouse), the same efforts with conditions comparable (group age) is required before claiming their limitation. VPA as a drug acts in cells (human, rat or marmoset) in the same way and then produces a large number of variations with a diffusing side effect. The variation in gene expression between species could easily be explained by differences not only in their origin but also in the 
conditions, timing of sample preparation and above all, the assays (array, RT-PCR or RNA-seq) for analysis. All the works referred to in the manuscript are not comparable in this respect and differences are naturally expected.

With phrases like:

“Genetically modified mouse models had even fewer concordant modules with human ASD. For example, the Fmr1 knockout mouse had an astrocyte related module that tended to be modulated in the same direction as human ASD, and the Mecp2 heterozygous mouse had a neuron/oligodendrocyte-related module modulated in the same direction with human ASD, but none of these modules were significantly concordant with human ASD."

It is completely expected that the effects of distinct genetic mutations are different and not at all comparable to all autism. As expected, these two examples referred in the discussion are simply not comparable to all autism and do not lead to a conclusion regarding autism in general.

As an additional comment on the presentation, it is not clear if the results are reproducible in every marmoset / autistic child one by one (100\%)? or if the results are variable (especially for gene expression), and if the average is presented? This question would be relevant for a generalization to autism in general, showing individual variation and not only means. 